MONIKA ZASOWSKA

University of Silesia in Katowice

monikazasowska@gmail.com

\title{
REVIEWS OF SINGLE-AUTHORED VERSUS MULTIPLE-AUTHORED ACADEMIC BOOKS. IS TWO LESS THAN ONE?
}

Keywords: academic book reviews, evaluation, academic values, value markers

\begin{abstract}
Although academic book reviews have been extensively discussed in a number of languages and in terms of a variety of factors, there is at least one point that has not yet been taken into consideration, namely the authorship factor (i.e. the number of the academic book authors) and its possible influence on evaluative language of the review. This assumption has given rise to the present study, which centres on a corpus-based analysis of one hundred linguistic book reviews with a half written by a single author and the other fifty being a collection of more than two authors. The investigation rests on Giannoni's (2010) typology of academic values, from which three values, i.e. GOoDNEss, NOvELTY and RELEVANCE and their lexical evaluative markers have been subjected to manual and automatic analyses with the aim to comparing and contrasting variation in value distribution in two corpora. Furthermore, the overall research findings have been presented in the form of the chi-square test in order to determine whether there exists any statistical significance between the selected categorical variables, and comment on accordingly.
\end{abstract}

\section{Introduction}

Extensive research into the study of personal opinion has proved how complex and diversified the phenomenon of evaluation may be and how linguistically challenging over the years it has become to produce a unanimous definition of the term itself. Being a matter of contention, this highly elusive and context-dependent aspect of language has been overlapped with a wide range of different concepts discussed under 
the heading "evaluative language" (see e.g. Conrad, Biber 2000; Hunston, Thompson 2000; Hyland, Tse 2004; Martin, White 2005; Bednarek 2006). The topic of evaluation may be therefore broadly understood within the frameworks of affective meaning, appraisal, stance, metadiscourse, or evaluation, to name only the most well-established terms. While this effectively means that some of them are closely synonymous, others cover subtly different areas of language of personal opinion (e.g. that of language and emotions) and as such require adopting a different approach that ultimately leads to the diversity in terminology. Essentially, what evaluation is, or what it is taken to be, is the underlying reason for all the conceptual differences that lie behind it.

By going beyond many of the traditional accounts, Martin and White (2005) proposed a semantic system of appraisal, which must be seen within the broad outlines of Systemic Functional Linguistics, from which it has emerged and of which it is a vital part. Martin and White (2005: 42) refer to "system of meanings" which are used by the speaker or writer to "approve and disapprove, enthuse and abhor, applaud and criticize, and (...) position their listeners/readers to do likewise". A large variety of language resources is directly connected to one of the basic tenets of SFL, i.e. the three metafunctions of language, out of which interpersonal meaning roughly corresponds to the general concept of evaluation. Investigating personal expression has also appeared under the label stance, which is, according to Conrad and Biber (2000: 57): "a cover term for the expression of personal feelings and assessments". In their corpusbased analysis of stance adverbials, Conrad and Biber (2000) differentiate between epistemic, attitudinal and style stance, each of which being differently used in three distinctive sets of corpora. For Hyland and Tse (2004: 157) metadiscourse is "the linguistic resources used to organize a discourse or the writer's stance towards either its content or the reader". Similarly, the element of subjective evaluation is present in Thompson and Hunston (2000: 5) where evaluation is "the broad cover term for the expression of the speaker or writer's attitude or stance towards, viewpoint on, or feelings about the entities or propositions that he or she is talking about". Seen in this light, evaluation could be considered as an action - something that a person does. As Hunston (2010) observes, this action may be performed in language and as such is amenable to linguistic investigation, or lie outside the study of language. Following Thompson and Hunston (2000), the action of why, when, how or what speakers and writers evaluate is also the approach of choice in the present paper.

As the sense of any academic research lies in its objective of searching for truth by refining and expanding our knowledge in the form of theories, evaluation as a form of assessment of such theories is a central and inevitable feature of almost all forms of academic discourse. It is perhaps best seen in review genres, which are produced with a view to providing examination and re-examination of almost all academic productions. The most recent studies on evaluative resources in review genres have been aimed at exploring different factors that may influence the language of reviews. The investigations have been undertaken by a number of linguists who looked at different aspects of forms of evaluation from disciplinary, crossdisciplinary, and cross-cultural perspectives. A study undertaken by Motta-Roth (1998) looked at the structure of academic book reviews in linguistics, economics, 
and chemistry. An analysis of politeness markers in book reviews in the area of linguistics was offered by Valor (2000), while Römer (2005) focused on the expression of negative evaluation in linguistics books to investigate the relations between gender and negative evaluation in The Linguist List reviews. For Tse and Hyland (2009) examining the construction of gender identities in book reviews revealed that there was no one-to-one correspondence between gender and the language used in the reviews. Diani (2009) dealt with reporting verbs (with special attention paid to argue and suggest) in book review articles across disciplines of linguistics, history, and economics to find any genre-oriented motivation behind evaluative commentary. The cross-cultural standpoint was taken in the studies by De Carvalho (2001) and Suárez-Tejerina (2005), who offered a contrastive analysis of Portuguese and Spanish book reviews, respectively, compared with the English book reviews. Other studies on the English-Spanish interface were undertaken by Suárez and Moreno (2006) or Salager-Meyer et al. (2007) and Lorés-Sanz (2012). An important observation was made by Lorés-Sanz (2012), who argued that book reviews in English displayed both praise and criticism, whereas Spanish book reviewers by avoiding negative criticism heavily loaded the reviews with praise. For English and Italian a similar examination was offered by Bondi (2005) and Giannoni (2006).

The following corpus-based and parameter-based study is yet another attempt at exploring language of evaluation in academic review genres. It is principally focused on linguistic academic book reviews, with special attention paid to the number of authors of the academic book under review. The interest in the authorship factor grew from an initial observation of strikingly different evaluative expressions found in the reviews of academic books written by one author and those being a collection of multiple authors. In order to examine the hypothesis which suggests that the academic book reviewers may intentionally use a different range of evaluative markers and/or lay more emphasis on different values in both subtypes of the book reviews, a corpus of one hundred reviews was compiled. A further important question to consider is whether there exists any systematic connection between the distribution of values and the authorship factor.

\section{Corpus and methodology}

As has been signalled above, this work has been based entirely on an analysis of two sets of corpora that consist of linguistic academic book reviews, each comprising fifty source texts published on The Linguist List in the years 2012-2013. For the sake of clarity, reviews of books with a single author and those being a collection of more than two authors are referred to as MONO and COLL respectively. The corpora are comparable in size - the total number of running words in MONO is 113,700 words and 132,478 words in COLL. All the files were converted into txt format and scanned with WordSmith Tools 6.0 (Scott 2012) for concordances of selected lexical items. The investigation rests on Giannoni's (2010) typology of academic values, but has been limited to three values, i.e. GOODNESS, NOVELTY and RELEVANCE, and their lexical markers. 
The primary way in which the present study accomplishes its objective is twofold. First, it is done through a quantitative analysis of the variation in value distribution in the two corpora. At this stage, of special interest is to determine which values come to the forefront in the reviews of a single author, and conversely, which are predominant in the reviews of multiple authors. Although part of the work was done automatically, a further stage of investigation required a thorough, manual inspection of each of the marker to ascertain that its evaluative meaning was preserved and remained relevant to the study. Throughout the paper examples are drawn from the source texts to illustrate evaluative markers in their original contexts. At this point it is worth noting that the present study is not aimed at differentiating between positive and negative forms of evaluation, as it purely concentrates on the analysis of evaluative marker distribution in the two corpora. However, as the instances of seemingly positive/negative evaluations were abundant, they could not be left completely unnoticed. As far as the organization of this part of study is concerned, the quantitative data are presented in tables, with the first column (MONO/COLL: TOTAL) showing the total number of occurrences of a selected value marker, and the second (MONO/ COLL: VALUE) presenting how many markers were found evaluative and, by extension, relevant to the analysis. The research findings are also shown on the bar graphs to give the overall picture of the distributional tendencies in the two corpora.

Second, a crucially important aspect of the following study manifests itself in the form of the chi-square test, a statistical tool essential to providing information on statistical significance of linguistic phenomena. It is the belief of the author that the result of it may enormously contribute to the statistical understanding of the matter.

That said, the figures obtained in the present study have been put into perspective and discussed in the final section of the paper.

\section{Values in academic settings}

If language of evaluation serves a purpose of reflecting an already established structure of norms - in other words - values of an academic community, it is essential to first look at them through the lens of humanities in general. Such a perspective is offered by Puzynina (1992: 83) according to whom evaluation is concerned with "stating what values and to what degree are characteristic of particular features, behaviour, states-of-affairs, and indirectly objects" (translation mine). In other words, what is being assessed could be located on a scale of positive or negative values within a particular content category. Her typology of values consists of the following thematic categories, each of which has a central concept as seen below:

- transcendental (metaphorical) values: sanctity/transcendental evil

- cognitive values: truth / ignorance and error

- aesthetic values: beauty/ugliness

- moral values: good of another person / harm of another person

- ethical values: propriety/inappropriateness

- vital values: life/death

- sensory values: happiness / unhappiness, pain 
Seen in this light, values can be analyzed from the philosophical, axiological, or psychological perspectives and accordingly cannot be unequivocally interpreted. Aiming to capture the values in their totality, Puzynina's classification proves too general and too inconsistent to be viewed from the perspective of academic discourse, particularly that of review genres. The above typology, as well as the overall approach to values and language is also challenged by Krzeszowski (1997: 16), for whom values are not just mirrored in language, but also "can be built into the perceptive apparatus, and hence into language". What is instead advocated is a much broader scope of axiology and linguistics combined together rather than a mere acknowledgment of the two disciplines.

It seems that a more adequate typology was proposed by Giannoni (2010), who identifies as many as twelve values prominently reported in the academic journals:

- goodness

- SIZE

- NOVELTy

- Relevance
- value

- TIMING

- IMPACT

- COMPlexity
- generality

- completeness

- centrality

- apPeal

Since much of academic activity centres on productions and publications, the above typology affords an opportunity to check whether certain, once-formulated academic criteria were fulfilled throughout the process of writing and in the final result of it. Thus, it proves to be working more effectively in the academic settings for all the parties concerned: writers, readers, and reviewers. As Hunston and Thompson (2000: 6) argue, "every act of evaluation expresses a communal value-system, and every act of evaluation goes towards building up that value-system" (italics mine). From this standpoint, it becomes clear that a value system gives rise to most, if not all, forms of academic activities and relationships. The range of evaluative resources is certainly of great importance but even more important seems to be the fact how these resources are used. This interest increases even more with a temptation to differentiate between two seemingly similar forms of academic book: a single-authored and a multiple-authored volume presented to the reviewer for a critical assessment.

\section{GOODNESS and GOODNESS markers}

In general terms, the concept of GOoDNEss corresponds to a well-known and almost intuitive distinction of good or bad. It is well reflected in the words of Hunston (1994: 191), who claims that "to evaluate something is to have an opinion about it, particularly in terms how good or bad it is". For Giannoni (2010: 89) GOodNEss is "a value that points to qualities that are viewed as positive or, conversely, as negative". This basic, but only superficially simplified distinction between good meaning positive on the one side of the scale, and bad meaning negative on the other, is also noticed by Krzeszowski (1997), who emphasizes its fundamental nature that manifests itself in both simplicity and complexity of use. The good (positive) - bad (negative) distinction is also more versatile in use, displays a much higher level of generality 
than other synonyms, e.g. beautiful or true, and in almost all situations translates into human experience. Thus, as Krzeszowski (1997: 14) observes, this scale proves to be an inherent aspect of categorization since "Man is a valuating being".

It is worth noting that different genres prioritize different values, and evaluations along different parameters. In the context of academic discourse, particularly that of academic review genres, the good - bad, or positive - negative parameter may not be necessarily the first to be applied, as there could be more emphasis placed on evaluation in terms of certainty or importance attached to the knowledge claim. That being said, however, the above does not undermine the good - bad distinction at all. According to Thompson and Hunston (2000), there is a strong, culturallydependent connection between certainty or importance and being good. In effect, the good - bad distinction turns out to be the most basic parameter of evaluation in discourse analysis to which all the other parameters relate.

As demonstrated by Giannoni (2010), the most prominent markers of Goodness fall into five groups, the majority of which are adjectives. The asterisk attached to some nouns and verbs indicates that they have been inspected in all their morphological variants.

- Group A. good, better, best, well, improve* |bad/poor, worse, worst, badly/poorly, worsen $^{*}$

- Group B. positive, positively| negative, negatively

- Group C. right, rightly|wrong, wrongly

- Group D. problem, problems, problematic, problematically, unproblematic

- Group E. error, errors, erroneous, erroneously

The lexical markers listed above have been subjected to the electronic and manual analyses, the quantitative data of which are presented below.

As Table 1 shows, the total number of GooDNEss markers identified in MONO and COLL amounts to 236 and 244 entries respectively, out of which 93 and 96 occurrences were evaluative and remained relevant to the study. This accounts for $39.4 \%$ and $39.3 \%$ of all cases respectively. At this point it is worth noting that the Group A markers fall into two sub-groups, each with a contrasting meaning to the other one, and it is the first sub-group (which centres solely on positive value markers) that is the most abundant in the entire corpus. By contrast, and perhaps surprisingly, the instances from the second sub-group markers were a rarity in both types of reviews.

As becomes evident from Table 1 the most predominant adjective is well, even though its evaluative meaning was preserved in a much smaller number of cases, i.e. 33 and 34 instances in both corpora. As has been signalled earlier, each item was thoroughly checked before counted as evaluative. Some basic reasons for exclusion usually concerned grammatical constructions, which, in the case of well, were many. Below are shown evaluative uses of well pertinent to the analysis:

- The book is well organized to include concise previews and summaries of each section. (MONO)

- For the most part, the papers were well written and well organized. However, certain problems remain. (COLL) 


\begin{tabular}{|c|c|c|c|c|c|c|}
\hline GOODNESS & $\begin{array}{c}\text { MONO: } \\
\text { total }\end{array}$ & $\begin{array}{c}\text { MONO: } \\
\text { value }\end{array}$ & Subtotal & $\begin{array}{c}\text { COLL: } \\
\text { total }\end{array}$ & $\begin{array}{l}\text { COLL: } \\
\text { value }\end{array}$ & Subtotal \\
\hline \multicolumn{7}{|l|}{ GROUP A } \\
\hline good & 34 & 20 & & 37 & 10 & \\
\hline better & 26 & 13 & & 37 & 19 & \\
\hline best & 23 & 19 & & 23 & 20 & \\
\hline well & 142 & 33 & & 133 & 34 & \\
\hline \multirow[t]{2}{*}{ improve } & 5 & 5 & & 6 & 6 & \\
\hline & & & $90 / 230$ & & & $89 / 236$ \\
\hline bad & 3 & 3 & & 0 & 0 & \\
\hline poor & 1 & 0 & & 4 & 3 & \\
\hline worse & 0 & 0 & & 1 & 1 & \\
\hline worst & 1 & 0 & & 1 & 1 & \\
\hline badly & 0 & 0 & & 0 & 0 & \\
\hline poorly & 1 & 0 & & 2 & 2 & \\
\hline worsen & 0 & 0 & $3 / 6$ & 0 & 0 & $7 / 8$ \\
\hline TOTAL & & & $93 / 236$ & & & $96 / 244$ \\
\hline
\end{tabular}

Table 1. Goodness markers in MONO and COLL: Group A

The second most frequent marker in this group is good, with 20 occurrences in MONO and 10 in COLL, which gives a significantly smaller number. The occurrences of good make for $21.5 \%$ and $10.4 \%$ in the two corpora respectively. Some of the contexts where good was found are:

- The author does provide some very good arguments for the application of speech genres (...) (MONO)

- This volume does a good job in balancing the variety and homogeneity of its content. (COLL)

Almost equal in size in both corpora is the number of better, best and improve. The typical contexts in which some of them occur are:

- This book is a must-read, as it gives a better understanding of the inner workings of the Japanese education system (...) (MONO)

- In truth, this is just one of the many features, outlined above, that set the book apart, and make it among the best such introductions in this category. (COLL)

As regards negative markers and what has already been mentioned, there are only rare instances of bad or poor, with three occurrences of the former in MONO and none in COLL. The situation is reversed in the case of poor, which occurs three times in MONO and only once in COLL. The other lexical markers such 
as worse, worst, badly, poorly or worsen are non-existent in MONO and only few instances of these are found in COLL. The instances of some less frequent markers are below:

- I believe that there are two reasons this kind of argument is a bad one. (MONO)

- I would like to mention just one more paper I found particularly compelling (...), which not only provides new insights into the semantic structure of nominal states, poorly covered in the existing literature, but also (...) (COLL)

Now, let us go to discuss the data gathered from Group B and Group C.

\begin{tabular}{lrrrrrr}
\multicolumn{1}{c}{ GOODNES } & $\begin{array}{r}\text { MONO: } \\
\text { total }\end{array}$ & $\begin{array}{c}\text { MONO: } \\
\text { value }\end{array}$ & Subtotal & $\begin{array}{r}\text { COLL: } \\
\text { total }\end{array}$ & $\begin{array}{c}\text { COLL: } \\
\text { value }\end{array}$ & Subtotal \\
\hline GROUP B & & & & & & \\
\hline positive & 17 & 15 & & 23 & 4 & \\
positively & 4 & 2 & 2 & 1 & \\
negative & 15 & 8 & & 23 & 5 & \\
negatively & 2 & 2 & $27 / 38$ & 5 & 1 & $11 / 53$ \\
\hline GROUP C & & & & & & \\
\hline right & 7 & 4 & & 17 & 2 & \\
rightly & 2 & 2 & & 1 & 1 & \\
rightfully & 0 & 0 & & 1 & 0 & \\
wrong & 4 & 4 & & 3 & 3 & \\
wrongly & 0 & 0 & $10 / 13$ & 0 & 0 & $6 / 22$ \\
\hline TOTAL & & & $37 / 51$ & & & $17 / 75$
\end{tabular}

Table 2. Goodness markers in MONO and COLL: Group B and C

The results in Table 2 reveal a disproportionate frequency of evaluative markers from the two groups in the two types of reviews: $72.5 \%$ and $22.6 \%$ respectively. The frequency of the most predominant evaluative adjective positive is significantly higher in MONO (88.2\%) than in COLL (17.3\%). As to its antonym, negative, the situation is similar. As far as these results are concerned, it is vital to note that there seems to be a clear preference for the use of lexemes positive/negative in the MONO-corpus than the other way round. The typical contexts are below:

- This narrative leads to positive learning experiences. (MONO)

- Empirical evidence is given much importance by all the authors, which is undoubtedly positive. (COLL)

- A slight negative point could be that, from time to time, the author seems to forget this didactic purpose (...) (MONO) 
- While many authors have called orthography in Early Modern times "erratic", "arbitrary" and even "chaotic", none of the authors in this volume have such a negative view of variation. (COLL)

Interestingly, Group $\mathrm{C}$ does not show such discrepancies, as right and wrong are distributed almost equally in the two corpora. Below are some contexts of these:

- There is nothing wrong with this in principle, but it sometimes seems to me that the use of stance is not very constrained (...) (MONO)

- I highly recommend this book as both a text for the classroom and a helpful starting point for fieldworkers needing to be pointed in the right direction for further study. (MONO)

A further analysis is based on the data obtained from Group D and Group E, which comprise exclusively negative lexical items.

\begin{tabular}{|c|c|c|c|c|c|c|}
\hline GOODNESS & $\begin{array}{c}\text { MONO: } \\
\text { total }\end{array}$ & $\begin{array}{c}\text { MONO: } \\
\text { value }\end{array}$ & Subtotal & $\begin{array}{c}\text { COLL: } \\
\text { total }\end{array}$ & $\begin{array}{c}\text { COLL: } \\
\text { value }\end{array}$ & Subtotal \\
\hline \multicolumn{7}{|l|}{ GROUP D } \\
\hline problem & 63 & 38 & & 55 & 30 & \\
\hline problems & 49 & 27 & & 46 & 18 & \\
\hline problematic & 17 & 17 & & 15 & 15 & \\
\hline problematically & 0 & 0 & & 0 & 0 & \\
\hline unproblematic & 1 & 1 & & 1 & 1 & \\
\hline
\end{tabular}

$83 / 130$

64/ 117

\begin{tabular}{|c|c|c|c|c|c|c|}
\hline GROUP E & & & & & & \\
\hline error & 4 & 2 & & 11 & 3 & \\
\hline errors & 43 & 29 & & 46 & 15 & \\
\hline erroneous & 0 & 0 & & 2 & 2 & \\
\hline \multirow[t]{2}{*}{ erroneously } & 0 & 0 & & 1 & 1 & \\
\hline & & & $31 / 47$ & & & $21 / 60$ \\
\hline TOTAL & & & $114 / 177$ & & & $85 / 177$ \\
\hline
\end{tabular}

Table 3. Goodness markers in MONO and COLL: Group D and E

As Table 3 indicates, in total there are exactly 177 occurrences of lexical markers in both corpora. After a manual inspection what remained relevant to the present investigation comprises 114 occurrences in MONO and 85 in COLL, which accounts for $64.4 \%$ and $48 \%$ respectively. An important observation at this point is related to a considerable difference in terms of the frequency of problem(s) and error $(s)$ in the two corpora. Both these words, and their plural forms, occur much more frequently in MONO than in COLL, which amounts to $54.2 \%$ and $37.2 \%$ respectively. 
This, however, should not be definite proof of negative evaluation on the part of the reviewer. Of crucial importance here is to note that in many cases both problem(s) and error(s) were preceded by a negative determiner 'no' and a non-negated verb, which certainly does not accommodate a negative assessment. Some illustrations of Group D and E markers include:

- These errors and inconsistencies might be more easily overlooked (...) but there really is no excuse for the publication of so error-laden a book as this (...) (MONO)

- There are no typographical errors on any page and the book is very well produced. (MONO)

- The most disconcerting aspect of this new volume is the proportion of mistakes, which include errors of typography, inconsistency, and simple accuracy. (COLL)

- For the most part, the papers were well written and well organized. However, certain problems remain. (COLL)

The marker problematic is almost evenly distributed in both corpora, with 17 occurrences in MONO and 15 in COLL. Below are the typical contexts where it was used:

- The final issue I found to be particularly problematic was the author's analysis of "maeoki". (MONO)

- The last of these topics is problematic (...) (COLL)

Finally, although there are only two occurrences of evaluative unproblematic in the entire corpus, they are worthy of attention owing to the construction they form, i.e. litotes. Generally speaking, this figure of speech, also referred to as a double negation, may be used to weaken the strength of evaluation:

- The chapter builds on the (not unproblematic) assumption that TOT states slow down lexical access (...) (COLL)

Some rare instances of erroneous and erroneously are found only in COLL:

- As discussed earlier in this review, this is an erroneous consequence of the GCMT derived by Honeck, not one claimed by proponents of the GCMT.

\subsection{GOODNESS - results}

Now, let us have a look at the distributional tendencies in the entire corpus as shown in Figure 1. On the whole, there is an overwhelming preference for Group A and Group D markers in the two corpora. In the first case, it is the COLL corpus that slightly outnumbers the other. However, the situation is reversed in the second case, where markers are visibly preferred in the MONO corpus. The least numerous group is Group C, where the difference in distribution is generally small, while Group B and E are comparable in size, with a predominance of markers in the MONO corpus.

It is important to look at these results comprehensively and think of any possible reasons for one group being predominant over the other. It is especially seen 
in the case of Group A and C, the distribution of which varies greatly, even though they both can be seen comprising largely synonymous markers. On the basis of the results, it becomes evident that the good - bad distinction (Group A) dominates over a similar one, i.e. that of right - wrong (Group C) distinction. This may be an indicative of what has already been said about the nature of good - bad scale, i.e. that evaluations in terms of how good something is are usually the starting point of every assessment, being the most basic and intuitive of all the others. Such may be the case in the book reviews selected for this study.

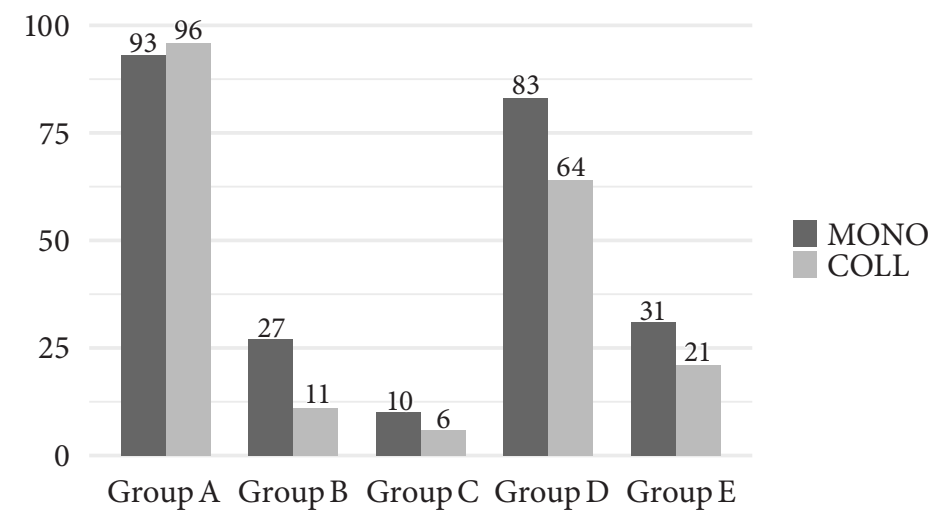

Figure 1. Distribution of GooDNEss markers in the corpora

As far as parts of speech are concerned, GoodNess is described mostly by adjectives and nouns, which form two largest group in the entire corpus (Figure 2). Both adjectives and nouns are more frequent in the MONO-corpus, while adverbs are distributed almost equally in both types of reviews. The least frequent group is that of verbs, which seems to be almost negligible as far as evaluations of GOODNESS are concerned.

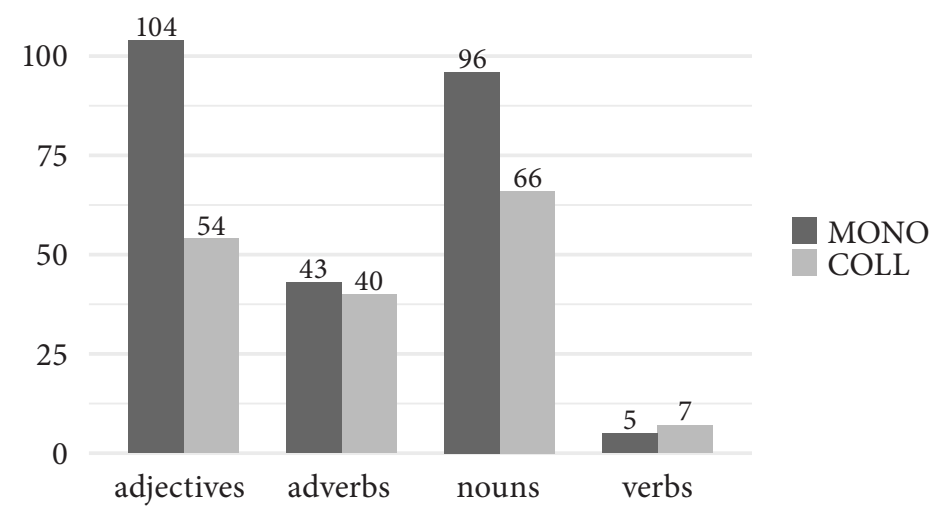

Figure 2. Parts of speech in GoodNEss 


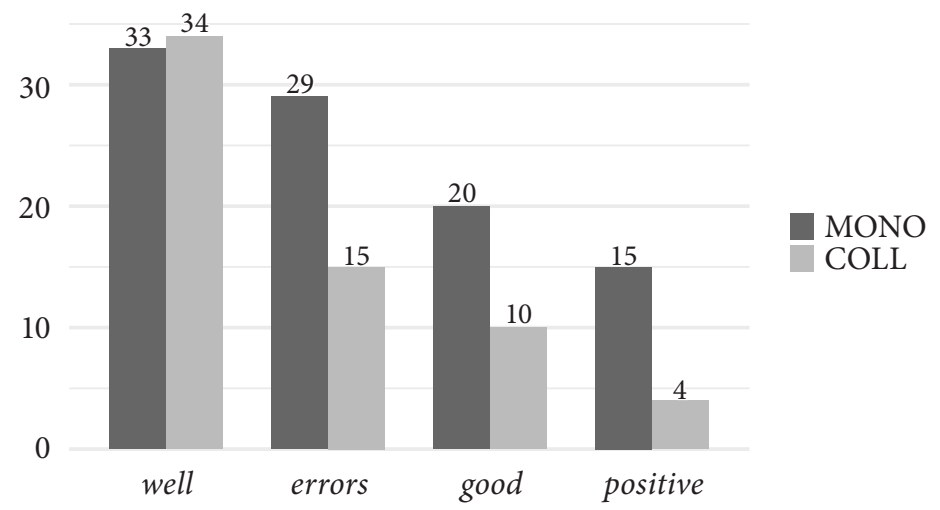

Figure 3. Most common evaluative markers of Goodness in the corpora

As has been signalled earlier and what Figure 3 now graphically shows, there is a clear preference for evaluative well in the entire corpus. The difference between MONO and COLL is minor, with 33 occurrences in MONO and 34 in COLL. The second most common evaluative marker is errors and it occurs almost twice as many times in MONO than in COLL. The same situation could be observed in the case good and positive - both are significantly more frequent in the MONO-corpus, with a fewer instances in the other corpus. All in all, the distribution of the individual evaluative markers, except for well, is at a consistently higher level in the MONO-corpus.

\section{Novelty and NOVelty markers}

For academic communities in general it is usually highly desirable for any research (whether it is conducted by an experienced and recognized author or a lesser-known novice) to occupy a previously untaken "research niche" and present the academic community with some new discoveries in the field (Swales 1990). This, in turn, could possibly evolve into formulating new theories which, following the notion of NovELTY, are validated by the reviewers. Therefore, NOVELTY may be readily considered a core value in any academic endeavor, as it indicates the condition of being new, which embraces the meanings of something fresh, original, or previously untackled. The concept of NOVELTY is hence linked with the condition of being new meaning something fresh, original, and not previously tackled.

Arranged by Giannoni (2010), the markers of NOveLty, which include mainly adjectives and adverbs, form three separate groups:

- Group A. new, newer, newly, novel, novelty, fresh |old, obsolete, antiquated

- Group B. innovate ${ }^{\star}$, innovation(s), innovative, renew ${ }^{\star}$, renewed

- Group C. recent, recently, latest

The detailed analysis of the above is presented in Table 4.

In total, there are 70 evaluative markers in MONO and 137 in COLL, which make up $33.4 \%$ and $60.1 \%$ respectively. Despite its predominance in the entire corpus, 


\begin{tabular}{|c|c|c|c|c|c|c|}
\hline NOVELTY & $\begin{array}{c}\text { MONO: } \\
\text { total }\end{array}$ & $\begin{array}{c}\text { MONO: } \\
\text { value }\end{array}$ & Subtotal & $\begin{array}{c}\text { COLL: } \\
\text { total }\end{array}$ & $\begin{array}{c}\text { COLL: } \\
\text { value }\end{array}$ & Subtotal \\
\hline \multicolumn{7}{|l|}{ GROUP A } \\
\hline new & 133 & 52 & & 123 & 76 & \\
\hline newer & 1 & 0 & & 1 & 1 & \\
\hline newly & 1 & 0 & & 1 & 1 & \\
\hline novel & 5 & 4 & & 9 & 4 & \\
\hline NOVELTY & 3 & 3 & & 0 & 0 & \\
\hline fresh & 1 & 1 & & 1 & 1 & \\
\hline old & 17 & 2 & & 20 & 2 & \\
\hline obsolete & 0 & 0 & & 1 & 0 & \\
\hline antiquated & 0 & 0 & & 0 & 0 & \\
\hline
\end{tabular}

$62 / 161$

$85 / 156$

\begin{tabular}{|c|c|c|c|c|c|c|}
\hline GROUP B & & & & & & \\
\hline innovate & 0 & 0 & & 0 & 0 & \\
\hline innovation/s & 4 & 3 & & 10 & 4 & \\
\hline innovative & 5 & 5 & & 11 & 8 & \\
\hline \multirow[t]{2}{*}{ renew $^{*}$} & 8 & 1 & & 0 & 0 & \\
\hline & & & $9 / 17$ & & & $12 / 21$ \\
\hline \multicolumn{7}{|l|}{ GROUP C } \\
\hline recent & 26 & 5 & & 35 & 30 & \\
\hline recently & 5 & 3 & & 10 & 7 & \\
\hline \multirow[t]{2}{*}{ latest } & 0 & 0 & & 4 & 3 & \\
\hline & & & $8 / 31$ & & & $40 / 49$ \\
\hline TOTAL & & & $79 / 209$ & & & $137 / 226$ \\
\hline
\end{tabular}

Table 4. Novelty markers in MONO and COLL

evaluative meaning of new was preserved in a smaller number of cases - 52 instances in MONO and 76 in COLL, which shows a relatively considerable difference between the two corpora. A similar thing can be observed with recent, the second most frequent marker in the group, whose evaluative use was most prevalent in the COLL corpus. Some illustrations of these markers are below:

- In sum, this book provides a new angle on a key concept (...) (MONO)

- Overall, this original contribution to the fields of (critical) sociolinguistics and literacy studies aligns with very recent attempts (...) (MONO) 
- In this new proposal, morphology is seen as a filter for which elements can be moved and where. (COLL)

- The first thing that stands out in the book is the large number of recent contributions included. (COLL)

The distribution of novel and innovative, as illustrated in the examples below, is fairly similar in both corpora, while there are no instances of obsolete and antiquated.

- Mickan's work is certainly an opportune addition to the literature on curriculum design and its use of tasks at the end of each chapter, although not innovative, does allow the reader to engage more fully with the ideas. (MONO)

- (...) they have been implemented to explore specific research questions, and fresh and innovative ways of thinking about the lexicon, bilingualism, and cognition. (COLL)

- This book proposes a novel theory of ellipsis licensing. (MONO)

\subsection{Novelty - results}

As becomes evident from Figure 4, NOVELTY markers are not evenly distributed in the two corpora. There is a strong preference for the COLL corpus as far as Group A markers are concerned. A similar situation can be observed in the case of Group C, which is again visibly preferred in COLL. Group B, however, does not show such striking differences and remains comparable in size. While the differences among all the three groups may be easily explained (it seems the markers themselves play a crucial role in their distribution, with new or recent being more readily used by the reviewers than, for instance, innovative), a more interesting observation occurs. As the data have shown, the value marker distribution between the two corpora may indicate a rather more conscious sensitivity to the notion of NOVELTY in the multipleauthored academic books than those written by one author only. The question that immediately arises is why the reviewers tend to seek and report on NOVELTY more intensively than they do with a single-authored book. Does it seem more urgent, desirable, or at least manageable to do so in a volume where often divergent ideas, approaches, or assumptions are scattered over several chapters than in a single author's volume where his/her viewpoint is more readily accessible, consistent and remains rather unchanged throughout the whole work? Although there is no immediate answer to that, this issue definitely merits further consideration.

Let us now move on to compare the part-of-speech distribution. As seen from Figure 5, in the entire corpus NOVELTY is consistently expressed by means of adjectives, yet the numbers in MONO stand in stark contrast to those in COLL, giving 69 and 125 occurrences in each. As far as adverbs and nouns are concerned, their distribution is almost even and almost equally negligible in the two corpora, with no similar discrepancy visible.

Figure 6 shows the most frequently occurring evaluative markers of NOVELTY, which are new and recent. Their distribution in the entire corpus varies greatly, especially in the case of the latter. In both situations it is the COLL-corpus that keeps the majority of these adjectives. 


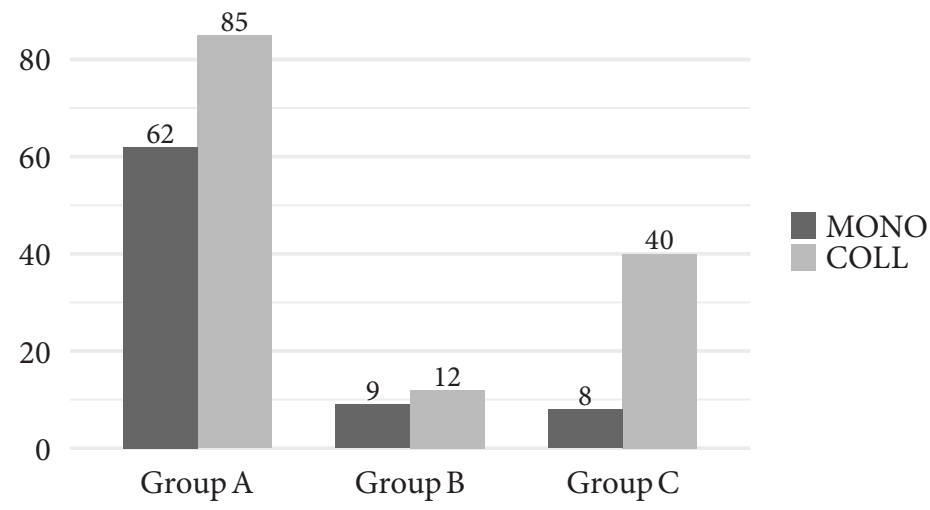

Figure 4. Distribution of NOvELTY markers in the corpora

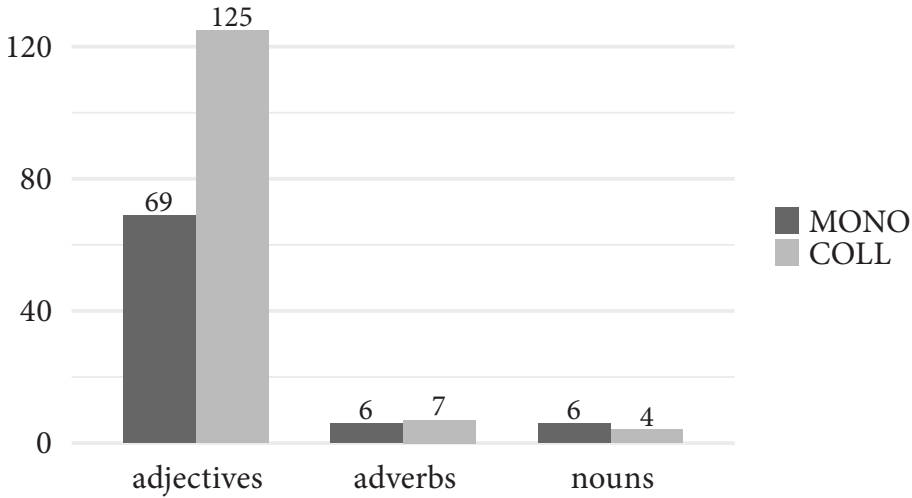

Figure 5. Parts of speech in NOvelTy

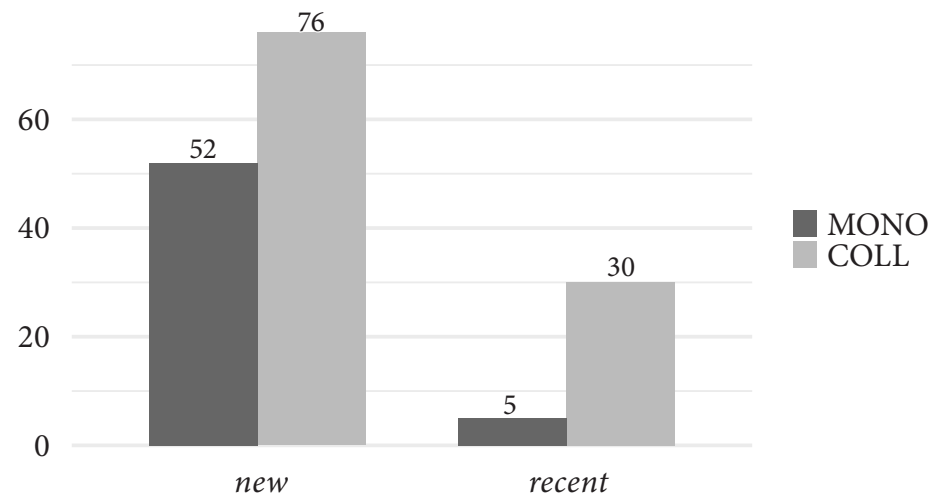

Figure 6. Most common evaluative markers of NOvELTY in the corpora 


\section{Relevance and relevance markers}

It is certainly through RELEVANCE that the reviewers can best assess the importance of academic productions and the extent to which these can contribute to the given area of study. In his work, Giannoni (2010) describes RELEVANCE and its wide range of lexical markers as a key aspect of the academic value system, as its main function lies in indicating the reader which parts of research deserve special attention from the reader. For Hunston (1994), who considers 'RELEVANCE' as one of the three functions of evaluative speech act, it is a purely analytical category, while for Thompson and Hunston (2000) 'RELEVANCE' is instrumental in organizing a text since instances of it are usually located at the very beginning or end of paragraphs/ sections. Bednarek (2006) considers "RELEVANCE" as one of the three news values that indicate the overall importance of news story for the news audience. According to Swales and Burke (2003), "RELEVANCE" is as a quality that shows a close relation of a thing under discussion to the field of study it relates to, an approach also seen in Thetela (1997), whose work on evaluative claims in research articles is also concentrated on how significant the research findings are. It is worth noting that Thetela's (1997) term of choice is "significance" and his view also incorporates the notion of interest, which for other researchers (see e.g. Swales and Burke 2003) is considered under a separate category, i.e. that of "assessment".

All these perspectives show that RELEVANCE revolves around adjectives denoting importance or significance and the like, primarily used when directing the reader towards the very essence of a text. In this study, lexical items describing RELEVANCE constitute the second largest group of all the three analyzed in this paper and are as follows:

- Group A. importance, important, importantly| unimportant

- Group B. major, main, principal, mainly, principally|minor

- Group C. significant, significance, significantly|insignificant

- Group D. relevant, relevance|irrelevant

- Group E. key, crucial, vital, crucially

Like in the case of GOODNESS and NOVELTY, RELEVANCE is expressed mostly by means of adjectives, which make up for nearly $61 \%$ of all the RELEVANCE markers in the study. As seen above, there are considerably fewer evaluative adverbs and nouns, with no instances of verbs taken into account. The quantitative analysis of RELEVANCE markers are presented in tables below.

In view of the results shown in Table 5, we see that both corpora present a similarly high number of evaluative NOvelTy markers in Group A and Group B - 178 occurrences in MONO and 164 in COLL, which makes for $67.9 \%$ and $65.3 \%$ respectively. The most frequent markers are important, main and importance. While the frequency of importance is equal in MONO and COLL, it is not the case of important, which is significantly higher in COLL. The situation is reversed with main, which is preferred in the MONO reviews. Instances of NOVELTY markers are presented below: 


\begin{tabular}{|c|c|c|c|c|c|c|}
\hline RELEVANCE & $\begin{array}{c}\text { MONO: } \\
\text { total }\end{array}$ & $\begin{array}{c}\text { MONO: } \\
\text { value }\end{array}$ & Subtotal & $\begin{array}{c}\text { COLL: } \\
\text { total }\end{array}$ & $\begin{array}{c}\text { COLL: } \\
\text { value }\end{array}$ & Subtotal \\
\hline \multicolumn{7}{|l|}{ GROUP A } \\
\hline importance & 38 & 30 & & 42 & 30 & \\
\hline important & 60 & 46 & & 80 & 58 & \\
\hline importantly & 11 & 11 & & 5 & 5 & \\
\hline unimportant & 1 & 1 & & 0 & 0 & \\
\hline
\end{tabular}

$88 / 110$

$93 / 127$

\begin{tabular}{|c|c|c|c|c|c|c|}
\hline GROUP B & & & & & & \\
\hline major & 33 & 21 & & 28 & 14 & \\
\hline main & 84 & 42 & & 52 & 30 & \\
\hline principal & 2 & 1 & & 5 & 4 & \\
\hline mainly & 24 & 18 & & 23 & 9 & \\
\hline principally & 0 & 0 & & 0 & 0 & \\
\hline \multirow[t]{2}{*}{ minor } & 9 & 8 & & 16 & 14 & \\
\hline & & & $90 / 152$ & & & $71 / 124$ \\
\hline TOTAL & & & $178 / 262$ & & & $164 / 251$ \\
\hline
\end{tabular}

Table 5. Relevance markers in MONO and COLL: Group A and B

- The book, even though reporting on the findings of a study conducted in New Zealand, is an important contribution to literature on (...) (MONO)

- Informed by both practice and theory, and indeed serving as a model for how one necessarily must feed the other, this volume is timely and deeply important. (COLL)

- It certainly fulfills the main aims of the book stated in the introduction (...) (MONO)

- One of the main points the authors talk about is a process they call "high-tone plateauing". (COLL)

As far as mainly is concerned, it is predominant in the MONO-corpus. Such is not the case with minor - it is more frequently used in the COLL reviews than in MONO:

- This volume is relevant mainly for those who are concerned with the dialogue of the two main traditions of contemporary philosophy. (COLL)

- One minor critical remark, which does not diminish the value of this volume (...) (MONO)

- One minor criticism of the book is the lack of careful editing work prior to publication. (COLL)

Major is yet another adjective considerably used in both corpora, with 21 occurrences in MONO and 14 instances in COLL. The less frequent markers are unimportant, and principally, with only one occurrence of the former in MONO and none of the 
latter in the entire corpus. The instance of principal are scarcely to be found both in MONO and in COLL.

- Such a question may, however, prove unimportant within a Construction Grammar framework (...) (MONO)

- The principal methods and findings of this work are (...) (COLL)

- Theoretically the book is a major contribution to Optimality Theory. (MONO)

- This volume makes a major contribution to our understanding of the approach to $(. .).(C O L L)$

Let us now move to the last two groups of RELEVANCE markers, which are shown below.

\begin{tabular}{lcccccc}
\multicolumn{1}{c}{ RELEvance } & $\begin{array}{c}\text { MONO: } \\
\text { total }\end{array}$ & $\begin{array}{c}\text { MONO: } \\
\text { value }\end{array}$ & Subtotal & $\begin{array}{c}\text { COLL: } \\
\text { total }\end{array}$ & $\begin{array}{c}\text { COLL: } \\
\text { value }\end{array}$ & Subtotal \\
\hline GROUP C & & & & & & \\
\hline significant & 14 & 12 & & 40 & 28 & \\
significance & 16 & 14 & & 11 & 8 & \\
significantly & 6 & 4 & & 8 & 5 & \\
insignificant & 0 & 0 & & 1 & 1 & $42 / 60$ \\
& & & $30 / 36$ & & & 42 \\
\hline
\end{tabular}

\begin{tabular}{lrrrr}
\hline GROUP D & & & & \\
\hline relevant & 47 & 40 & 61 & 48 \\
relevance & 13 & 8 & 28 & 14 \\
irrelevant & 1 & 0 & 3 & 1
\end{tabular}

$48 / 61$

$63 / 92$

\begin{tabular}{|c|c|c|c|c|c|c|}
\hline \multicolumn{7}{|c|}{ GROUP E } \\
\hline key & 35 & 28 & & 34 & 28 & \\
\hline crucial & 14 & 10 & & 22 & 16 & \\
\hline vital & 1 & 1 & & 8 & 3 & \\
\hline \multirow[t]{2}{*}{ crucially } & 7 & 7 & & 6 & 5 & \\
\hline & & & $46 / 57$ & & & $52 / 70$ \\
\hline TOTAL & & & $124 / 154$ & & & $157 / 222$ \\
\hline
\end{tabular}

Table 6. Relevance markers in MONO and COLL: Groups C-E

As Table 6 shows, there are 124 evaluative markers RELEVANCE in MONO, which gives $80.5 \%$ and 157 occurrences of these COLL, which makes up $70.7 \%$ of all. One of the most predominant adjective is relevant, with the frequency slightly higher in COLL than in MONO. The same concerns relevance, which is once more preferred in COLL. The typical contexts in which relevant occurs are: 
- However, the book is equally relevant here. (MONO)

- A concluding chapter presenting concrete pedagogical implications obtained from the studies would thus have complemented the discussions making this volume relevant to a larger audience, including teachers. (COLL)

- It would seem that the relevance of this paper is lost due to its positioning in the volume. (COLL)

Another frequent adjective is $k e y$, which is equally distributed in both corpora:

- The volume explores the key area of motion as well as the lesser-studied concepts of definiteness and number. (MONO)

- Most importantly though, is that here Reuland establishes key points on which later discussion is based, Chapter 2, Provocation, gives the key proposal of the book. (COLL)

What Table 6 also reveals is a noticeable predominance of the evaluative adjective significant in the COLL-corpus, which was used in a context such as :

- Chapter 2 contains significant explanation of CFL and conventional phraseology. (COLL)

As far as significance is concerned, it is not evenly distributed in both corpora, with a clear preference in MONO. An illustration of it is seen, for example, in:

- The significance of the findings of the fifth chapter is that (...) (MONO)

Crucial and crucially are not consistently distributed, with the former being more frequent in COLL, while the latter in MONO. Some contexts are:

- Crucial is the distinction between conventional meanings and implicated meanings. (MONO)

- This is a crucial chapter. (COLL)

- More crucially however, Cinque barely addresses the conceptual problems that have been raised for this kind of account. (MONO)

- This relatively dense chapter crucially emphasizes the need to understand these new techniques (...) (COLL)

The less frequent markers in both corpora are insignificant, irrelevant and vital:

- They point to problems with studies that have low statistical power; that is the effects observed may in fact be due to an insignificant sample size which leads to null result. (COLL)

- With the rapid development of various historical corpora, the book will soon be far from exhaustive in that respect, but even so it should not become irrelevant. (COLL)

- This chapter gives insights into this vital area. (COLL) 


\subsection{ReLeVANCE - results}

It is evident from Figure 7 that the most frequent markers are those in Group A and $\mathrm{B}$ as opposed to the markers from the other groups. In almost all cases, except for Group B, the markers of RELEVANCE are predominant in the COLL corpus.

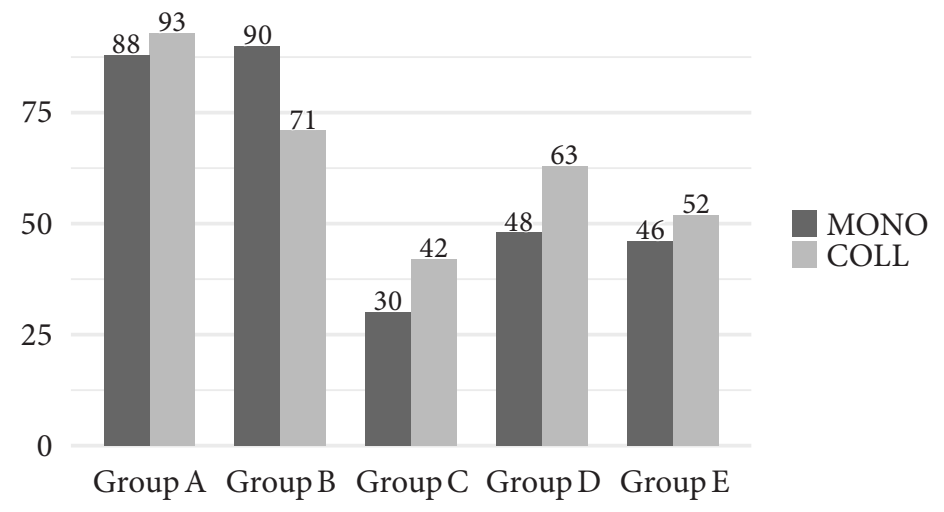

Figure 7. Distribution of RELEVANCE markers in the corpora

As regards parts of speech, Figure 8 shows a strong preference for adjectives in both corpora, with a predominance of them found in COLL (245 occurrences) than in MONO (210 occurrences). By contrast, and perhaps not surprisingly, adverbs were significantly less frequent in both corpora, while the number of nouns is identical.

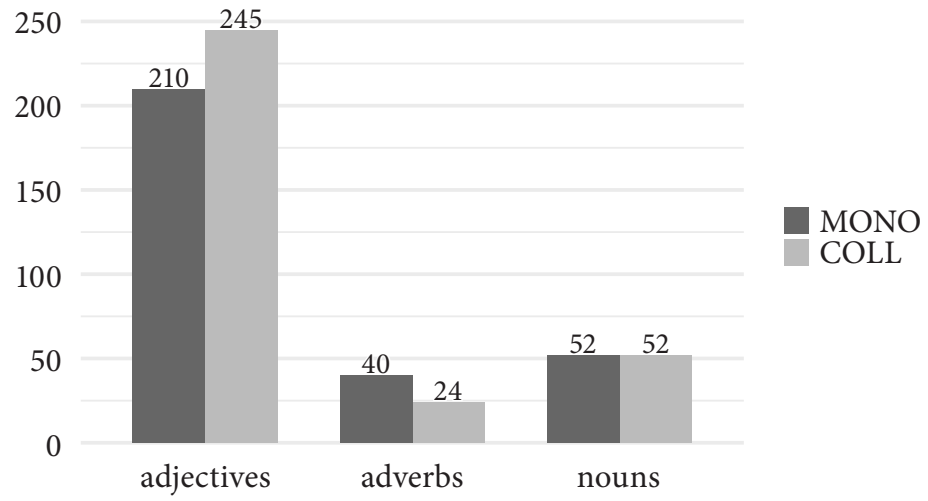

Figure 8. Parts of speech in RELEVANCE

The investigation has also shown that the vastly preferred markers of RELEVANCE are important, relevant, main, and key. As far as important and relevant are concerned, the distribution is significantly higher in the COLL corpus, but it is definitely not a general tendency for the rest of the markers under consideration. In favour of the MONO corpus is main, while key is distributed equally in both corpora. 


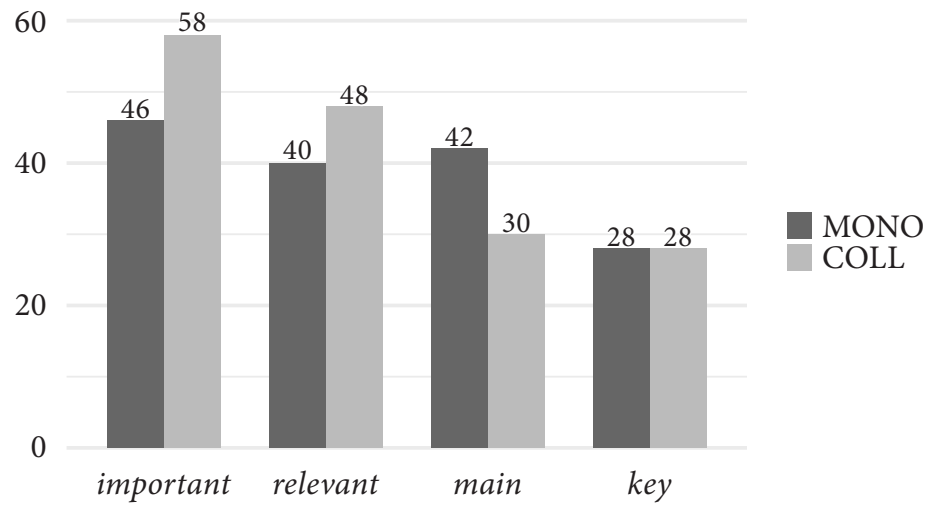

Figure 9. Most common evaluative markers of RELEVANCE in the corpora

An interesting observation that emerges from the data is the fact that greater emphasis of important and relevant is placed on the academic books being a collective volumes than these of a single author. The question whether it is a systematic undertaking on the part of the reviewers to direct their and the readers' attention mostly towards important and relevant parts of the collective volumes remains open. A possible explanation can again be sought in the diversity of topics collected in the volume of multiple authors than that of a single author.

\section{The chi-square test}

Yet another crucially important aspect of the present study manifests itself in the form of the chi-square test, a statistical tool for measuring relationship between selected categorical variables. According to Butler (1985), it is particularly useful in situations where one wishes to check whether a set of selected data fits to a mathematical model (and if so, to what extent), or in cases where one wishes to check whether two characteristics are independent or associated with one another, in a way that high frequencies of one tend to be related with high frequencies of the other, which is the case in this study of value and the authorship connection.

The two characteristics are the MONO and COLL reviews being investigated in the paper. On the null hypothesis, there is no association between the frequency of the three academic values and the authorship type. To test the hypothesis, the chi-square test has been performed with the data shown in Figure 10. The contingency table (Table 7) provides the following information: the observed cell totals, (the expected cell totals) and the chi-square statistic for each cell.

The chi-square test has revealed that the chi-square statistic is 25.8204 , with the result being significant at $p<.05$. For this reason, the null hypothesis must be rejected, as there is a systematic relationship between the two factors taken into account, i.e. that of value and that of the number of authors. In light of the above, it may be stated that the authorship factor affects the overall evaluation in each of the corpora. 


\begin{tabular}{l|rr|l} 
& \multicolumn{1}{|c|}{ MONO } & COLL & \multicolumn{1}{c}{ Row Totals } \\
\hline GOODNESS & $244(214.05)[4.19]$ & $198(227.96)[3.94]$ & 442 \\
NOVELTY & $70(100.25)[9.13]$ & $137(106.76)[8.57]$ & 207 \\
RELEVANCE & $302(301.70)[0.00]$ & $321(321.30)[0.00]$ & 623 \\
\hline Columns - Total & 616 & 656 & 1272 (Grand Total)
\end{tabular}

Table 7. The chi-square results (obtained by means of Chi-Square Calculator)

Although the entire corpus chosen for the study may be regarded as not fully representative, the limitation of which the author is well aware, it is worth noting that even such a sample corpus has proved to show a statistical significance indicating an association between values and the number of authors of the book under review. There are grounds, then, for saying that this corpus-based study has shown some pattern which otherwise might have remained concealed.

\section{Discussion and concluding remarks}

In the light of the overall findings, it behooves us to reflect on some tentative conclusions drawn from both the quantitative observations and the statistical perspective in the study.

First and foremost, as Figure 10 shows, the results indicate not consistently balanced distribution of evaluative lexical markers in the MONO and COLL reviews.

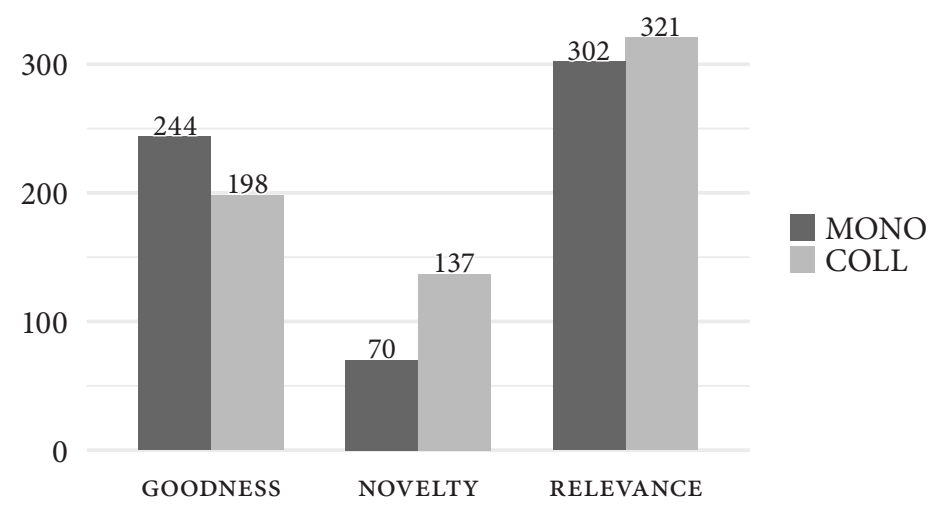

Figure 10. Distribution of GoODNESS, NOVELTY and RELEVANCE in the corpora

It is clearly seen that the three values investigated in this study are distributed with a different frequency, the highest frequency having been found for RELEVANCE and the lowest for NOVELTY. The markers of RELEVANCE were distributed almost equally in the two corpora, yet with a slight predominance in COLL: 302 occurrences in MONO 
and 321 in COLL. In the case of GOODNESs, the second most frequent group, a clear predominance is seen in MONO: 244 occurrences there and 198 in the COLL corpus. As far as NOVELTY is concerned, there is a wide discrepancy in the two corpora, with a strong preference for NOVELTY markers in the COLL corpus, the fact which might be an indicative of the reviewers' conscious decision rather than mere coincidence to prioritize NOVELTY in the collective volume.

Second, it is necessary to take into account the nature of both types of reviews and the possible impact of the strict guidelines set by The Linguist List, where all the reviews must take a pre-determined form and be approximately similar in length. Given a relatively fixed structure and a limited space of the review section, the reviewers must then work out a strategy for comprehensive evaluation of multi-chapter and multiauthored volumes. In effect, a detailed evaluation of each of the chapter is practically impossible. Besides, there is a tendency to evaluate a COLL volume more as a whole rather than a collection of individual articles. These factors joined together may also influence language of evaluation, and by extension, the value markers of choice.

Third, a predictable yet still worthy of mention aspect of the study is the distribution of parts of speech throughout the entire corpus (Figure 11).

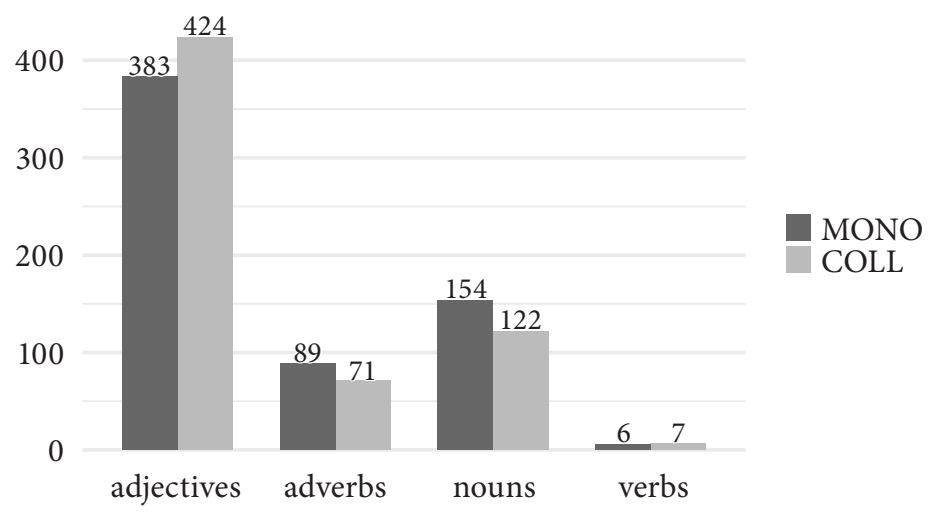

Figure 11. Parts of speech in the corpora

It is perhaps of lesser importance to argue that the value markers of GOODNEss, NOVELTY and RELEVANCE were mostly signalled by means of evaluative adjectives, as the number of adjectives itself was the highest in each of the group considered. Instead, what is worth noting is that in both MONO and COLL corpus the differences in the distribution of all the four parts of speech are generally comparable. In total, and to which there is no surprise, the most common evaluators are adjectives: 383 occurrences in MONO and 424 in COLL. Both adverbs and nouns were distributed more frequently in MONO, while the frequency of verbs was rather unexpectedly low.

Last but not least, although much of the work conducted in this paper draws on quantitative information and, to a lesser extent, qualitative interpretation, the statistical analysis performed in the last section has offered fresh insights into how language of academic book evaluation works. That being said, the chi-square result 
may spark interest in the authorship factor and be used in a broader way, i.e. that of academic discourse in general.

All in all, while it is beyond the scope of this study to provide a more comprehensive look at evaluation and authorship, it is the hope of the author that the findings presented and discussed above have shed some light on a previously unexamined topic. With various forms of academic productions on the rise, in print and online, further investigations into the possible correlation between language of evaluation and the number of authors in the context of review genres and beyond is most welcome and needed, as it promises a potentially new path for linguistic research to follow.

\section{References}

Bednarek M. 2006. Evaluation in media discourse. New York, London.

Bondi M. 2005. Metadiscursive practices in academic discourse: Variation across genres and disciplines. -Bamford J., Bondi M. (eds.). Dialogue within discourse communities. Metadiscursive perspectives on academic genres. Tubingen: 3-29.

Butler Ch. 1985. Statistics in linguistics. Oxford.

Conrad S., Biber D. 2000. Adverbial marking of stance in speech and writing. - Hunston S., Thompson G. (eds.). Evaluation in text: Authorial stance and the construction of discourse. Oxford: 56-73.

De Carvalho G. 2001. Rhetorical patterns of academic book reviews written in Portuguese and in English. - Iglesias L. Rabade, Doval Suarez S.M. (eds.). Studies in contrastive linguistics. $2^{\text {nd }}$ International Contrastive Linguistics Conference. Santiago de Compostela: 261-268.

Diani G. 2009. Reporting and evaluation in English book review articles: A cross-disciplinary study. - Hyland K., Diani G. Academic evaluation. Review genres in university settings. Basingstoke: $87-104$.

Giannoni D. 2006. Expressing praise and criticism in economic discourse: A comparative analysis of English and Italian book reviews. - Del Lungo Camiciotti G., Dossena M. (eds.). Variation in business and economic discourse. Diachronic and genre perspectives. Rome: 126-138.

Giannoni D. 2010. Mapping academic values in the disciplines. A corpus-based approach. Bern. Hunston S. 1994. Evaluation and organization in a sample of written academic discourse. Coulthard M. (ed.). Advances in written text analysis. London: 191-218.

Hunston S. 2010. Appraising research. Evaluation in academic writing. Basingstoke.

Hunston S., Thompson G. 2000. Evaluation in text: Authorial stance and the construction of discourse. Oxford.

Hyland K., Tse P. 2004. Metadiscourse in academic writing: A reappraisal. - Applied Linguistics 25.2: 156-177.

Krzeszowski T.P. 1997. Angels and devils in hell. Elements of axiology in semantics. Warszawa. Lorés-Sanz R. 2012. Local disciplines, local cultures: Praise and criticism in British and Spanish history book reviews. - Brno Studies in English 38.2: 97-116.

Martin J.R., White P.R.R. 2005. The language of evaluation. Appraisal in English. Basingstoke. Motta-Roth D. 1998. Discourse analysis and academic book reviews: A study of text and disciplinary cultures. - Fortanet I., Posteguillo S., Palmer J.C., Coll J.F. (eds.). Genre studies in English for academic purposes. Castelló de la Plana: 29-58. 
Puzynina J. 1992. Język wartości. Warszawa.

Römer U. 2005. This seems somewhat counterintuitive, though... Negative evaluation in linguistic book reviews by male and female. - Tongini-Bonelli T., Del Lungo Camiciotti G. (eds.). Strategies in academic discourse. Amesterdam: 97-115.

Salager-Meyer F., Alcaraz Ariza M.A., Pabón Berbesí M. 2007. Collegiality, critique and the construction of scientific argumentation in medical book reviews: A diachronic approach. - Journal of Pragmatics 39.10: 1758-1774.

Scott M. 2012. WordSmith tools version 6. Stroud.

Suárez L., Moreno A.I. 2006. The rhetorical structure of academic journal book reviews: A cross-linguistic and cross-disciplinary approach. [Online document retrieved from https://www.unizar.es/aelfe2006/ALEFE06/1.discourse/28.pdf].

Suárez-Tejerina L. 2005. Is evaluation structure-bound? An English-Spanish contrastive study of book reviews. - Tongini-Boneli E., Del Lungo Camiciotti G. (eds.). Strategies in academic discourse. Philadelphia.

Swales J.M. 1990. Genre analysis. Cambridge.

Swales J.M., Burke A. 2003. It's really fascinating work: Differences in evaluative adjectives across academic registers. - Leistyna P., Meyer Ch. (eds.). Corpus analysis. Language structure and language use. Amsterdam: 1-18.

Thetela P. 1997. Evaluated entities and parameters of values in academic research articles. English for Specific Purposes 16.2: 101-118.

Thompson G., Hunston S. 2000. Evaluation: An introduction. - Hunston S., Thompson G. 2000. Evaluation in text: Authorial stance and the construction of discourse. Oxford: 1-27.

Tse P., Hyland K. 2009. Discipline and gender: Constructing rhetorical identity in book reviews. - Hyland K., Diani G. (eds.). Academic evaluation: Review genres in university settings. London: 105-121.

Valor G.M.L. 2000. A pragmatic approach to politeness and modality in the book reviews articles. València. 
